\title{
Creación de materiales didácticos digitales y uso de tecnologías por parte de los docentes de Primaria. Un estudio de casos
}

\author{
Isabel María Gallardo Fernández ${ }^{1}$ \\ Raquel Mariño Fernández ${ }^{2}$ (D) \\ Ana Vega Navarro ${ }^{3}$ (D) \\ ${ }^{1}$ Universidad de Valencia (UV), España; ${ }^{2}$ Universidad de Santiago de Compostela (USC), España; ${ }^{3}$ Universidad de La \\ Laguna (ULL), España
}

Resumen. Este artículo pretende analizar cómo la creación y usos de materiales didácticos digitales, por parte de los docentes, potencia un cambio metodológico en las aulas de Primaria. Para ello, se ha realizado un análisis de documentos y webs de tres estudios de caso, entrevistas semiestructuradas con los diferentes agentes y observación de las aulas de cuarto, quinto y sexto de Educación Primaria de tres Comunidades Autónomas (Canarias, Galicia y Valencia). Los resultados muestran que los docentes abogan por la creación de sus propios materiales, con el fin de adecuarlos a las características de cada grupo-clase y diseñar una propuesta de enseñanza contextualizada y que potencie la formación de sujetos críticos y creativos. Estamos ante un tema complejo que demanda para la escuela del Siglo XXI una nueva reconversión digital en sus profesionales, espacios y currículos.

Palabras clave: Educación Primaria; material didáctico digital; investigación cualitativa; tecnología educativa; desarrollo profesional docente.

Criação de materiais didáticos digitais e o uso de tecnologias por professores dos anos iniciais do Ensino Básico. Um estudo de casos

Resumo. Este artigo visa analisar como a criação e o uso de materiais didáticos digitais, por professores, promove uma mudança metodológica nas salas de aula do Ensino Básico (Fundamental). Para este fim, foi realizada uma análise de documentos e websites de três estudos de caso, entrevistas semiestruturadas com diferentes agentes e observação em sala de aula de turmas do quarto, quinto e sexto ano, em três Comunidades Autônomas (Canárias, Galiza e Valência). Os resultados mostram que os professores defendem a criação de seus próprios materiais, com o fim de adaptá-los às características de cada turma e de elaborar uma proposta de ensino contextualizada, que favoreça a formação de sujeitos críticos e criativos. Estamos diante de um tema complexo que exige para a escola do século XXI uma nova transformação digital em seus profissionais, espaços e currículos.

Palavras-chave: Ensino básico/fundamental; materiais didáticos digitais; pesquisa qualitativa; tecnologia educacional; desenvolvimento profissional.

Creation of digital teaching materials and use of technologies by Primary teachers. A case study Abstrat. This article aims to analyze how the creation and use of Digital Teaching Materials by teachers promotes a methodological change in Primary classrooms. For this, an analysis of documents and websites of three case studies, semi-structured interviews with the different agents and observation of the fourth, fifth and sixth Primary classrooms of three Autonomous Communities (Canary Islands, Galicia and Valencia) has been carried out. The results show that teachers advocate the creation of their own materials, in order to adapt them to the characteristics of each group-class and design a contextualized teaching proposal that enhances the formation of critical and creative subjects. We are facing a complex issue that demands for the school of the XXI century a new digital reconversion in its professionals, spaces and curricula.

Keywords: Primary Education; digital didactic material; qualitative research; educational technology; professional development. 


\section{Introducción}

El presente trabajo se enmarca en el proyecto de investigación $L a$ escuela de la sociedad digital: análisis y propuestas para la producción y uso de los contenidos digitales educativos ${ }^{1}$. A continuación, presentamos unas pinceladas del estado del arte en el tema que nos ocupa para centrarnos en tres estudios de casos: Canarias, Galicia y Valencia.

Las Tecnologías de la Información y Comunicación (TIC) fueron y son las principales causantes de la revolución digital. Esta revolución permanece en un estado evolutivo y activo, generando nuevas formas, usos y códigos de comunicación y gestión de la información en nuestras sociedades. Ante esta realidad, la escuela se ve inmersa en un profundo cambio que demanda nuevos enfoques en sus metodologías y materiales educativos. El diseño y organización de los materiales didácticos del siglo XXI, exige incorporar los nuevos aportes digitales a los diversos escenarios de la vida para que sean más interactivos, constructivistas, innovadores, multimedia, hipertextuales y pedagógicos (Area, 2017; Bautista, Martínez e Hiracheta, 2014; George, 2020).

Nuestras escuelas han de adecuar su discurso y prácticas educativas a esta realidad social, sin perder su esencia principal como entidades generadoras de procesos de enseñanza y aprendizaje, significativos y relevantes (Duffy y Jonassen, 2013; Pérez-Gómez, 2012). El cambio e innovación pedagógica necesarios para transformar estos espacios educativos, conlleva someter a debate sus estructuras organizativas, didácticas-curriculares, profesionales y culturales asistiendo a nuevas fórmulas organizativas del trabajo (Weller, 2016). Ya no basta con sustituir el soporte impreso por otro digitalizado, se necesita ofertar mayor formación docente en competencias tecnológicas y usos pedagógicos. Esto requiere dotar a los centros educativos con mejores recursos, materiales, contenidos, herramientas y servicios para incentivar nuevas medidas reglamentarias que legislen todo el proceso (Area, 2020; De Pablos, 2015; Cepeda, Gallardo y Rodríguez, 2017).

Para la infancia y la juventud actual, las TIC se han convertido en objetos habituales en su vida y en señas de identidad generacional que los distingue de los adultos. Estos jóvenes nacidos en la última década del siglo XX son la primera generación socializada bajo la cultura digital y una escuela digitalizada. Para ellos, es algo cotidiano incorporar los recursos digitales a sus rutinas de aprendizaje en el aula, por lo que los docentes hemos de

Proyecto Escuel@ Digital, la escuela de la sociedad digital: análisis y propuestas para la producción y uso de los contenidos digitales educativos, (EDU201564593-R), financiado por el Plan Estatal de I+D+i. I.P.: Manuel Area Moreira. 
ofrecerles espacios y procesos educativos más dinámicos, participativos e innovadores (Area, Parcerisa y Rodriguez, 2010; San Martín y Peirats, 2018; Vidal, Vega y López, 2019).

Asimismo, la educación del presente conlleva una socialización y concienciación cultural y moral, que se transmite de generación en generación y que depende, en parte, de la sociedad en la que una persona se educa (Peirats, Gallardo, San Martín y Cortés, 2015). Además, tiene un alcance global digitalizado, porque está presente en todas nuestras acciones e interacciones con las tecnologías, dentro y fuera del espacio educativo. De hecho, son varios los estudios (Arboleya y Dopico, 2017; García-Martín y Cantón-Mayo, 2019) que evidencian cómo los países tecnológicamente desarrollados, muestran como indicador de calidad educativa a las escuelas que trabajan para acabar con la brecha digital. Una brecha que sigue muy arraigada en aquellos sectores sociales más vulnerables, discriminados y con menores recursos socioeconómicos, técnicos y de carácter productivo. Surge así la necesidad de legislar la utilización de las tecnologías en las aulas, como medio a través del cual ejecutar el proceso educativo, tal como queda recogido explícitamente en los Art. 111.5 y Art 111.6 de la Ley Orgánica para la Mejora de la Calidad Educativa (LOMCE, 2013).

Las tecnologías proporcionan un aporte que mejora el proceso de enseñanza y aprendizaje, siempre y cuando sean utilizadas como un apoyo y no como un reemplazo del propio proceso docente (enseñanza) y/o del estudio autónomo (aprendizaje). Asumimos con Area (2017), que la escuela del siglo XXI necesita reinventar su praxis y su material didáctico con un enfoque de mayor calado, más revolucionario y con un papel pedagógicamente más innovador. Sabemos que pasar del soporte papel al soporte digital conlleva una transformación profunda en el marco social, normativo y educativo, que va más allá del proceso de enriquecer o añadir hiperenlaces y vídeos a los textos escritos.

Toda esta riqueza y diversidad, digital y tecnológica, conforman nuestro campo de estudio que, en mayor o menor medida, ya están presentes en nuestras realidades educativas (González, Martín y Vega, 2018).

\section{Métodos y materiales}

La finalidad principal de este artículo es dar respuestas al planteamiento del problema: cómo la creación y usos de material didáctico digital, por parte de los docentes, potencia un cambio metodológico en las aulas de cuarto, quinto y sexto de Primaria. La metodología global seguida 
en el proyecto Escuel@ Digit@।, responde a un estudio mixto que combina métodos cuantitativos y cualitativos a lo largo de cuatro fases (López-Roldán y Fachelli, 2015).

Dichas fases se concretan en cuatro estudios de distinta naturaleza, tanto en sus objetivos como en sus procedimientos y ámbito de actuación. El Estudio / centró su interés en explorar y conocer las características pedagógicas y mapa de la oferta de portales, webs y plataformas educativas, gestionadas por las diferentes administraciones y editoriales en el territorio español. El Estudio // buscó identificar las representaciones de los agentes y actores implicados en el diseño, difusión y utilización de los contenidos digitales educativos. El Estudio /// reúne los estudios de casos para conocer el uso real de los contenidos digitales en centros y aulas de Educación Primaria. El proyecto se cerró con el Estudio IV que, a partir de los resultados obtenidos, elaboró una guía de buenas prácticas sobre la producción, distribución y usos de los MDD.

\subsection{Contexto y propósito de la investigación}

Este artículo toma como referente el Estudio III del Proyecto Escuel@ Digit@| y centra el objeto del trabajo en analizar cómo la creación y usos de MDD, por parte de los docentes, potencia un cambio metodológico en las aulas de cuarto, quinto y sexto de Primaria de tres centros de Canarias, Galicia y Valencia. Para ello planteamos algunos interrogantes: ¿En qué aspectos la tecnología impulsa y genera cambios e innovaciones en los procesos de enseñanza aprendizaje? ¿Cómo la creación de MDD potencia un cambio metodológico en las aulas de cuarto, quinto y sexto de Primaria? ¿Qué formación necesita el profesorado de Primaria para implementar las tecnologías en las aulas? ¿Qué estrategias usa el profesorado para integrar las tecnologías en las aulas de Primaria del siglo XXI?

Pretendemos responder a dos objetivos generales:

- Estudiar la creación y el uso de Materiales Didácticos Digitales en tres estudios de caso de tres comunidades autónomas, que responden a tres realidades diferentes con diversos matices, visiones y enfoques.

- Analizar como la creación de MDD, por parte del profesorado, potencia un cambio metodológico en las aulas de cuarto, quinto y sexto de Primaria. 
Asimismo, se concretan los siguientes objetivos específicos:

- Reconocer que MDD utiliza el profesorado en Primaria, atendiendo a las asignaturas y al proceso de enseñanza y aprendizaje.

- Indagar sobre los recursos, elementos y agentes presentes en el proceso de creación de los MDD.

- Identificar las acciones y factores que potencian/favorecen un cambio metodológico en las aulas de cuarto, quinto y sexto de Primaria.

- Analizar qué tipo de formación necesita el profesorado de Primaria para implementar las tecnologías.

\subsection{Participantes}

Como población objeto de estudio se ha tenido en cuenta la opinión y valoración aportada por los Coordinadores TIC de los tres estudios de caso, así como por el profesorado que forman parte de los equipos directivos y los tutores y tutoras de aulas de cuarto, quinto y sexto de Primaria. En total fueron 22 entrevistas realizadas ( 11 en Canarias, 6 en Galicia y 5 en Valencia), apoyadas a su vez en 39 observaciones de aula (11 observaciones en Canarias, 16 en Valencia y 12 en Galicia).

Las entrevistas se han realizado de forma individual a cada docente. Las grabaciones en audio tienen una duración aproximada de entre 40 y 90 minutos. Asimismo, se ha realizado la transcripción literal de cada una de ellas, para la elaboración de las matrices que constituyen el elemento de análisis. Las observaciones de aula se han realizado durante las sesiones magistrales de los propios sujetos entrevistados y con una duración variable de 45 a 90 minutos cada una.

La muestra que conforma este artículo la integran tres centros, que pertenecen a tres comunidades autónomas y realidades educativas distintas, pero que pueden ser identificables con otras realidades y centros educativos del Estado español.

\section{Caso 1: Canarias}

Nos encontramos ante un centro concertado, situado en la Comunidad Autónoma de Canarias, en concreto en La Laguna de Tenerife. Es un centro semiurbano que oferta las etapas de educación Infantil, Primaria y Secundaria. Durante el curso 2017-2018, atendió aproximadamente a 1000 discentes y con un total de 68 docentes. 
El centro se seleccionó por las siguientes razones: titularidad privada concertada; el alumnado de $4^{\circ}$ y $5^{\circ}$ de Primaria utilizaba de modo habitual tabletas o iPads; varios docentes de $4^{\circ}$ y $5^{\circ}$ creaban MDD; y, el profesorado de $4^{\circ}$ y $5^{\circ}$ estaba dispuesto a ser observado y entrevistado. La realización de este estudio ha requerido el análisis de documentos institucionales, la realización de entrevistas y la observación en aulas de $4^{\circ}$ y $5^{\circ}$ de Primaria.

Cuatro notas caracterizan al centro: promueve metodologías innovadoras; es un centro bilingüe certificado; tiene una política institucional de implantación de las TIC; y, en Primaria, apuestan por el uso de tecnologías y pedagogías innovadoras tal como se refleja en el Plan Estratégico de Innovación Educativa. El centro tiene un coordinador de innovación desde hace dos años. Entre sus funciones están coordinar al equipo de innovación e impulsar el diseño y desarrollo del Plan de Innovación. Este centro ha supuesto una gran ayuda para la juventud de este entorno, pues el nivel socioeconómico de sus habitantes en esta zona está marcado por el origen humilde de sus ciudadanos. En cuanto al profesorado, el centro tiene un equipo docente bastante sólido y consolidado.

\section{Caso 2: Galicia}

En el caso de Galicia es un centro público de carácter semiurbano, con una trayectoria tecnológica creciente y consolidada en los últimos veinte años. Este centro pertenece al ayuntamiento de Teo, provincia de A Coruña, y escolariza alumnado procedente, en su mayoría, del medio rural próximo. El contexto en donde se ubica combina una actividad agrícola y ganadera con otros trabajos más vinculados con los sectores de la construcción y servicios.

Cuenta con un total de 20 unidades, 5 para Educación Infantil y 15 para Primaria. En la actualidad escolarizan un total de 389 niños/as: 104 de Infantil y 285 de Primaria. En cuanto a su equipo docente, lo forman 32 docentes, incluidas la jefa del departamento de Orientación, un maestro de inglés, un especialista de audición y lenguaje y otro de pedagogía terapéutica, compartidos con tres unitarias del Ayuntamiento de Santiago de Compostela. El centro tiene una Asociación de Madres y Padres del Alumnado (AMPA) muy activa y comprometida con la integración de las tecnologías en la vida comunitaria del centro.

La introducción en las tecnologías en este centro se inició en el año 2000 y continúa creciendo y progresando hasta la actualidad. Como momentos clave en materia de innovación tecnológica y trabajo con MDD, destaca el entrar a formar parte del Proyecto Abalar (2013), para digitalizar 
el centro, suprimir los libros de texto en formato impreso en beneficio de materiales digitales o por proyectos alternativos, así como, años después, en el curso 2014-2015 formar parte del proyecto Edixgal.

\section{Caso 3: Valencia}

Nos situamos en un centro urbano, de titularidad pública y en el contexto de un barrio de la ciudad de Valencia. Al igual que los dos casos anteriores, en él se imparten las etapas de educación Infantil y educación Primaria. En la actualidad cuenta con 221 discentes y 17 docentes. La ratio es de 26 discentes por curso, con excepción de educación Infantil de 3 años y cuarto curso de Primaria, donde existe reducción por alumnado con Necesidades Educativas Especiales.

En cuanto a la trayectoria innovadora del centro destaca su planteamiento de escuela inclusiva y el uso de las tecnologías en todos los niveles de la enseñanza. Además de que la metodología implementada en las aulas es por tareas y proyectos. Asimismo, la educación emocional del alumnado y el desarrollo de sus potencialidades personales y habilidades sociales son ejes básicos del trabajo en esta escuela. La Asociación de Madres y Padres del Alumnado forma parte activa en la vida del centro. De hecho, las familias han facilitado la incorporación de las TIC en las aulas. También, el centro está implicado en un proyecto experimental autonómico para impulsar el uso de los libros de texto en tabletas digitales en los cursos de quinto y sexto de educación Primaria. El centro participa en el proyecto Tabletas en centros educativos desde el curso 2012-2013, motivo por el que el alumnado de quinto y sexto dispone de una tableta por persona en la que tienen acceso a MDD.

\subsection{Procedimientos y recursos utilizados}

Siguiendo a autores como Flick (2004), Stake (1998), Simons (2011), Taylor y Bogdan (1984), la metodología elegida para este trabajo ha sido el estudio de caso, al considerarla como la más adecuada para investigar en profundidad y estado natural la realidad diaria de un centro.

Las observaciones de aula, junto con las entrevistas, nos han permitido identificar experiencias y actividades educativas que suponen la implementación de estrategias docentes (Angrosino, 2012). Hemos utilizado una parrilla de observación ya diseñada en el Estudio II del Proyecto Escuel@ Digital. 
En la organización y tratamiento de la información hemos tomado como referente el Informe de cada estudio de caso, así como la transcripción de las entrevistas que nos han servido para elaborar las matrices de análisis, estableciendo categorías y dimensiones (Tabla 1).

Tabla 1. Categorías y dimensiones de análisis

\begin{tabular}{ll}
\hline \multicolumn{1}{c}{ Categorías } & \multicolumn{1}{c}{ Dimensiones } \\
\hline Las TIC generadoras de cambio/innovación aulas & - Valoración General \\
& - Programación y MDD en el aula \\
\hline Creación de MDD en Primaria & Modelo de uso \\
& - Cambio metodológico en las aulas de $4^{\circ}$, \\
& $5^{\circ}$ y $6^{\circ}$ de Primaria \\
\hline Formación del profesorado & Necesidades formativas \\
& - Desarrollo profesional docente \\
\hline Estrategias a implementar en Primaria & - Integración TIC \\
& - Comunicación del centro con las familias \\
& - Estrategias utilizan/usan el profesorado
\end{tabular}

Fuente: Elaboración propia

\section{Resultados}

Afrontamos el análisis de resultados tomando como referencia las siguientes categorías: a) Las TIC como generadoras de innovación en las aulas; b) Creación y uso de MDD en Primaria; c) Formación del Profesorado; $y$, d) Estrategias docentes a implementar.

\subsection{Las TIC como generadoras de innovación en las aulas}

En relación a los cambios e innovaciones, en el Caso I (Canarias), observamos que el centro ha desarrollado una clara política de implantación de las TIC articulada en torno a: la dotación de recursos tecnológicos; la creación de puestos dedicados a las TIC; y, el uso de una plataforma que sirve para la gestión administrativa y la comunicación con las familias. El equipo directivo ha creado las condiciones para la implantación intensiva de las TIC y la promoción de su uso pedagógico. Sin embargo, el centro no dispone de Plan TIC.

Desde la implantación de Infantil y Primaria el colegio decidió prescindir del aula de informática. Siguen el modelo 1:1. Los dispositivos pertenecen a las familias y los pueden ir pagando poco a poco. No se pueden 
sacar del centro, son para uso académico. Cada aula de Primaria dispone de una torre, un proyector, una PDI con posibilidad de conexión a Apple Classroom, y altavoces, y acceso a internet por wifi. El centro ha adquirido recursos TIC al participar en un proyecto de formación del profesorado promovido por la Consejería de Educación consiguiendo así dotaciones para equipamientos tecnológicos.

El colegio dispone de servidor propio, una web oficial y dos blogs. También tiene cuentas institucionales en Twitter, YouTube y Google que gestiona el responsable de Comunicación.

Las características pedagógicas más relevantes de Primaria son:

- El uso intensivo de tabletas digitales. Los libros de texto se utilizan solo en algunas asignaturas.

- La existencia de superaulas. El alumnado de cada nivel se ubica en un aula separada por puertas plegables traslúcidas que se repliegan según trabajen por grupo-clase o por nivel.

- El desarrollo de la metodología de aprendizaje basado en proyectos en la jornada de la tarde en las materias Natural Sciences, Social Sciences, Visual Arts, y Lengua Castellana y Literatura, así como el aprendizaje cooperativo en prácticamente todas las asignaturas.

- Los grupos interactivos como experiencia piloto.

En el Caso 2 (Galicia), todas las aulas cuentan con un ordenador de sobremesa y un portátil, una pizarra digital, proyector, netbooks, mesas y sillas móviles, buena conexión wifi y materiales impresos complementarios.

En las aulas de quinto y sexto todo gira en torno a los espacios virtuales de aprendizaje (EVA) promovidos por el proyecto Edixgal. Esta iniciativa de la Consejería de Educación pretende instaurar el uso de los libros digitales y consolidar la digitalización de los centros educativos. Las diferentes materias impartidas en el aula se gestionan a través de netbooks en donde alumnado, docentes y familias, interaccionan con contenidos curriculares provistos por la editorial Edebé, la empresa de contenidos digitales Netex y los MDD creados por los docentes y otros profesionales. Estos espacios son muy polivalentes porque les permiten gestionar sus propios recursos y actividades, tanto docentes como discentes, así como el acceso a más de 2.900 recursos educativos digitales. La revolución tecnológica promovida 
por el Proyecto Abalar supuso instaurar una perspectiva de cambio a nivel institucional, personal y de equipo, así como diseñar su propio Plan TIC para gestionar, planificar y dinamizar dichos cambios.

En este centro se pasó de hacer un uso reducido y/o puntual de las TIC en las horas de informática, a integrarlas en todas las dinámicas y acciones vinculadas con los procesos de enseñanza y aprendizaje de las aulas de Primaria. Para visibilizar todo este proceso se crea y diseña una web propia, promovida por el Proyecto Webs Dinámicas (impulsado desde la Consejería de Educación), y se articula una intranet para favorecer la comunicación entre profesorado y familias.

Como evaluación global de los recursos y la infraestructura disponibles en el centro, destacan y valoran la evolución positiva que han vivido en estos últimos veinte años, incrementando su dotación económica y formativa gracias a un planteamiento que consistió, básicamente, en: poner plazos cortos y encontrar vías administrativas legales -paralelas- para lograr financiación, formación y participación directa de las familias.

En el Caso 3 (Valencia), para analizar en qué aspectos las TIC han sido generadoras de innovación en las aulas, hemos realizado un análisis de la documentación del centro, observaciones de aula en quinto y sexto de Primaria, una entrevista grupal con las familias y cinco entrevistas a los docentes (equipo directivo, coordinador TIC, coordinador de ciclo y profesorado de quinto y sexto de Primaria).

El centro está implicado en el Programa experimental autonómico para impulsar el uso de los libros de texto en tabletas digitales en quinto y sexto de Primaria, desde el inicio del Plan experimental con TIC de la Conselleria de Educación de Valencia (Resolución de 10 de junio de 2013) durante los cursos escolares 2013-2014 y 2014-2015.

Los recursos humanos del centro han sido un aspecto clave para realizar este proceso de integración de las TIC. En palabras de la directora: "la mayoría de los docentes teníamos clara la necesidad de aunar el concepto de qué era para nosotros ser escuela, en relación con el nuevo proyecto y las proyecciones futuras. Las tecnologías nos facilitan librar esa barrera entre instituciones formales, no formales e informales. Por coherencia con este concepto también adquirimos el compromiso de formación continua del claustro" (Valencia, entrevista directora). 
El proceso de gestión de los contenidos digitales en este centro escolar ha sido analizado en investigaciones previas por el Grupo CRIE, así como la implementación de los dispositivos digitales en las aulas (Peirats, Gallardo, San Martín y Cortés, 2015).

Como evaluación global hemos de destacar el liderazgo del equipo directivo en relación al impulso de las TIC. De hecho, han pedido la ampliación de la red wifi del centro: trabajan con dispositivos móviles (tabletas) desde que la Conselleria de Educación convocó el programa experimental, y debido a que el máximo que ofrece la administración sólo son $10 \mathrm{Mb}$, el AMPA del centro ha tenido que actuar e implicarse para resolver el problema. Esta mejora ha supuesto la posibilidad de poder trabajar todos con las tabletas.

\subsection{Creación y uso de MDD en Primaria}

En el Caso 1 (Canarias), encontramos un profesorado que combina el uso de diversos recursos tecnológicos, con los libros de texto digitales elaborados por las editoriales, objetos digitales de aprendizaje y/o entornos didácticos digitales, abiertos y/o privados.

Los docentes elaboran sus propios materiales, o los adaptan, empleando aplicaciones tales como PDF, Word, PPT, Popplet, Webquest 0 Blogs, entre otros. También usan Google como herramienta de búsqueda de contenidos, información y materiales que sirvan como recursos educativos. Todos han facilitado el trabajo en equipo y la comunicación, acortando las barreras espaciales y temporales y diversificando la información y conocimientos al alcance de su alumnado.

La función pedagógica y educativa de los medios digitales son un eje vertebrador del proceso de enseñanza-aprendizaje y se complementan o apoyan con otros materiales didácticos. Enriquecen y estimulan el proceso de enseñanza-aprendizaje, fomentan el papel activo y protagonista del alumnado y favorecen el uso de metodologías más motivadoras para estos. Como factores que han favorecido esa integración y usos destacamos:

- Una buena dotación de recursos tecnológicos, un servidor propio y dispositivos adecuados en cada aula. Reorganizaron sus recursos para incentivar un uso intensivo de las TIC en el profesorado y alumnado de Primaria.

- Los dos últimos directores impulsaron su integración pedagógica y organizativa, así como reforzaron el personal de ayuda: coordinador TIC y personal de apoyo tecnológico, un coordinador 
de innovación que promueve el uso pedagógico de las TIC, y un responsable de comunicación social.

- Decidieron no utilizar el libro de texto impreso y optar por un modelo 1:1 en toda la etapa de Primaria, promoviendo un uso de las TIC casi exclusivo por parte de todo el alumnado.

- El trabajo por proyectos en algunas materias también ha favorecido que sus prácticas educativas integren patrones metodológicos de aprendizaje por descubrimiento guiado; una mayor coordinación docente, planificación e intercambio de MDD y otros recursos.

- Tienen una imagen/identidad digital definida y una web amigable con perfiles en Facebook, Twitter y canal de YouTube. También participan en la red de centros de la congregación, beneficiándose del apoyo en el uso de la plataforma QualitasEducativa.

En el Caso 2 (Galicia), las maestras entrevistadas afirman que el mayor cambio metodológico y de usos didácticos que promueven las TIC en sus aulas, es que hacen evidente fomentar un aprendizaje descentralizado donde el alumnado debe aprender con y sin los docentes presentes, dentro y fuera del aula, con herramientas TIC o sin ellas. Consideran primordial lograr un cambio en la forma de entender e implementar los procesos de formación y aprendizaje.

Defienden que la integración y usos de las TIC en el aula, permiten una mayor apertura del currículo y del centro al entorno. Consideran prioritario que la comunidad educativa entienda que se puede aprender en cualquier situación y que el proceso de aprendizaje no termina en la institución escolar. Apuestan por un enfoque metodológico más contextualizado y conectado con la realidad de su alumnado, así el aprendizaje se vuelve más significativo (Galicia, maestro y director).

También hacen referencia a que lo importante es promover diseños y usos basados en el beneficio que aportan, y no en la obligatoriedad de integrarlos para digitalizar un proceso impuesto por las administraciones y/o sociedad. Cuando aluden a los beneficios de crear e integrar las tecnologías y MDD en el proceso global de enseñanza-aprendizaje, afirman que permiten ampliar los horizontes educativos si se abordan transversalmente desde el currículo (Galicia, maestra $6^{\circ}$ ).

Otra mejora metodológica es la transformación del proceso formativo en un proceso más atractivo, motivador e interesante para el alumnado, a la vez que proporciona a las familias concebirlas como unas herramientas ligadas a valores positivos, y alejadas del simple uso como entretenimiento, 
distracción y/o peligro. Consideran que es necesario contemplar las TIC en el Proyecto Educativo y Curricular del Centro como un valor más que empapa y contagia cualquier dinámica, acción y espacio educativo (Galicia, maestra de $5^{\circ}$ ). Como equipo docente tratan de buscar el mejor enfoque metodológico, didáctico y pedagógico en el diseño de MDD, para perfeccionar el aprendizaje competencial o funcional.

En el Caso 3 (Valencia), para dar respuesta a esta categoría nos fijamos en la coordinación, apoyo y asesoramiento para la integración pedagógica de las TIC en el centro. La coordinación y comunicación entre los docentes de quinto y sexto es un aspecto muy relevante, pues el apoyo entre profesionales surge en todas las asignaturas y momentos del proceso de enseñanza-aprendizaje.

La directora caracteriza la aportación de las TIC a la enseñanza y el aprendizaje desde una perspectiva de cambio institucional, personal, de grupo y de equipo puesto que "comportan nuevos métodos, nuevas maneras de trabajar, nuevos modos de atender a las familias, y de relacionarse entre los agentes educativos" (Valencia, directora). Este hecho implica también cambios en la gestión de aula, la dinamización, las pedagogías y las didácticas. Se trata de pasar a integrar las tecnologías en la dinámica de los procesos de enseñanza-aprendizaje en las diferentes aulas (Valencia, maestra de 69. Destacan que el "trabajar en equipo también ha supuesto romper con el horario de tutorías y priorizar a las personas ante los papeles. Si quieres construir Proyecto toda la comunidad y el equipo docente tiene que sentirse integrado, valorado, querido" (Valencia, directora).

El equipo directivo insiste en la necesidad de trabajar el concepto aprender entre el conjunto de docentes y discentes puesto que consideran que aprender es una formación que se lleva a cabo a lo largo de toda la vida. Se trata de ir más allá de una institución. Por lo tanto, desde la escuela debe fomentarse esa necesidad de aprender con y sin los docentes, dentro y fuera de la escuela. Asumen que el alumnado aprenderá en un futuro "muchas más cosas que aún no están ni inventadas" por lo que aquí "la institución acompaña" para proporcionar autonomía. Del mismo modo, se hablará de innovación si se logra "cambiar la manera de entender la formación y el aprendizaje" (Valencia, maestra de $5^{\circ}$ ).

\subsection{Formación del Profesorado}

Caso 1 (Canarias), para este centro la principal formación que debe tener su profesorado es saber cómo utilizar esas aplicaciones que se les enseña al alumnado. En primer lugar, consideran necesario formarse en 
como planificar con eficacia las sesiones interactivas, para así poder calcular el tiempo y una buena ejecución de la actividad digital diseñada. También consideran fundamental, formarse en competencias pedagógicas que permitan mejorar las programaciones didácticas, así como en técnicas para formular y prefijar objetivos didácticos a conseguir con el uso de estas aplicaciones y tareas digitales.

Todo docente debe tener una formación básica para dominar la gestión, diseño e implementación de los recursos y MDD dentro de las aulas, Pero sobre todo destacan la necesidad de formarse en la adquisición de habilidades y destrezas vinculadas con el desarrollo de la capacidad de empatizar con alumnado de escasa edad o con necesidades educativas especiales. Es decir, en el supuesto caso de encontrarse con un discente que le cueste entender y adquirir las competencias digitales, se hace necesario dedicarle unos momentos de atención más individualizada, así como comprender que quizás nunca haya usado o visto nada parecido en su entono sociofamiliar.

El centro cuenta con personal bien formado y dedicado a las TIC. Tiene tres coordinadores TIC, uno de Infantil y Primaria, otro de ESO y Bachillerato, y otro de Ciclos Formativos y FPB. EI coordinador TIC de Infantil y Primaria, se encarga de la formación tecno-pedagógica de ambas etapas.

52 Entre sus funciones están la coordinación con el equipo TIC del centro, y la administración del control de cuentas de usuarios y contratos. El colegio dispone de dos técnicos informáticos que realizan el mantenimiento de las redes y el hardware. Además, disponen de un Plan de Comunicación Social y de un responsable para dinamizarlo.

En el Caso 2 (Galicia), sobre la formación recibida y demandada por el profesorado, el equipo directivo y coordinador TIC consideran que en el centro siempre ha existido una preocupación por ampliar y mejorar la formación del profesorado en el uso y diseño de las TIC. Matizan que no les obsesionan los medios digitales, simplemente los usan e integran en la vida del aula por su valor y potencial didáctico; añadiendo que deben mejorar la formación del profesorado en la creación de MDD y manejo de nuevas metodologías. Comentan que su profesorado tiende más a adaptar los materiales ya existentes, que a crear otros nuevos porque no se siente seguro en el proceso. Consideran que lograr esa mejora formativa repercutirá en una mejora del trabajo colaborativo entre docentes, al motivarlos a crear sus propios materiales.

En cuanto a la oferta formativa indican que existen tanto cursos impartidos, gestionados y regulados por la propia Administración educativa, como otros no oficiales que son ofertados y realizados por el propio centro, según las demandas y necesidades de sus docentes, o en redes de apoyo 
intercentros, más informal y colaborativa. Ahora que ya están más formados y cualificados, gestionan la oferta para ampliar y mejorar su capacitación en TIC, optimizando el uso y manejo curricular de las mismas dentro del aula.

Tanto el coordinador TIC como el dinamizador, también consideran necesario actualizar su Plan TIC, elaborado hace más de 10 años por lo que muchos de sus planteamientos ya están obsoletos. Otra demanda formativa pendiente es la de mejorar las competencias para un uso más interactivo de las TIC, menos conductista e individualizado, y que proporcione una retroalimentación más activa en el proceso de enseñanza-aprendizaje del alumnado. Los contenidos formativos que este centro demanda como prioritarios para su profesorado serían: software libre; creación de recursos a través de diferentes herramientas como procesadores de texto, editores de imágenes, de páginas web, aplicaciones de maquetación, presentaciones multimedia; aplicaciones de apoyo a la diversidad (manejo de aplicaciones para el alumnado con necesidades especiales o dificultades de aprendizaje); y, transversalidad (propuestas de trabajo multidisciplinares).

En el Caso 3 (Valencia), siguiendo el Plan General Anual del centro, los planes de formación del curso 2017-2018 son de carácter mixto, acordes a las necesidades y demandas de formación que presenta su profesorado. Entre sus cursos destacan el seminario sobre Tratamiento de la imagen y sonido para la confección de recursos y MDD.

Respecto a la competencia y formación del profesorado para el uso pedagógico de las tecnologías, el Coordinador TIC comenta que todo el profesorado realiza formación en seminarios y grupos de trabajo con otros equipos docentes del contexto valenciano que están implicados en proyectos con tecnologías, diseño y creación de MDD, experiencias y redes educativas online (Valencia, coordinador TIC). Este profesorado asume que los contenidos de enseñanza pueden encontrarse en diversas fuentes y en internet, por lo que crean contextos de aprendizaje que desarrollan las distintas competencias.

El seminario de Formación basado en el tema de competencias y desarrollo del currículum, les ha permitido comprobar que el currículum, en su práctica, es más heterogéneo de lo que su imagen oficial presenta. En este sentido, los docentes debaten sobre la función de la escuela, los materiales curriculares y el papel del libro de texto en los procesos de enseñanza-aprendizaje. En sus prácticas diarias potencian situaciones de aprendizaje para el desarrollo de aptitudes y competencias mediante metodologías activas: diseño de proyectos, resolución de problemas, estudio de casos y simulaciones, entre otras (Valencia, maestra de $6^{\circ}$ ). 
Una de las dos docentes es considerada como la experta y figura de referencia en el centro, pero ambas tutoras de quinto y sexto destacan la necesidad de la autoformación para dar respuesta a las demandas que puedan surgir en el aula. Para ello, acuden al Centro de Formación, Innovación y Recursos Educativos (CEFIRE) de Valencia, para realizar cursos/formación, pero también para buscar tutoriales o información en línea. "Nuestra formación ha sido muy autodidacta y dedicando muchas horas (...) se trata de probar, experimentar y aprender" (Valencia, maestra de $5^{\circ}$ ). Podemos afirmar que en este centro "mientras enseñan, continúan aprendiendo" (Freire, 1997, p.10).

\subsection{Estrategias docentes a implementar}

En el análisis de esta categoría tomamos como referente a María Moliner (1998) que define estrategia docente como un proceso y/o plan organizado, flexible e intencional que persigue la consecución de unas metas didácticas preestablecidas. Su puesta en práctica conlleva, por parte del docente, una planificación del proceso de enseñanza-aprendizaje para el logro de unos objetivos.

Asumimos que todo proceso educativo debe integrar estrategias que fomenten aprendizajes significativos y relevantes. Tras esta delimitación conceptual mostramos las estrategias docentes que se implementan en los tres estudios de caso, tomando como referencia las observaciones de aula y las entrevistas realizadas.

En el Caso 1 (Canarias), se observa que en cada aula se emplean distintos medios digitales, como fue el caso de las sesiones de Lengua y Religión de $4^{\circ}$, en las que se utilizó la aplicación digital de LeoBien, además del libro de texto digital, con las que se hicieron diversas actividades en la pizarra digital y con los iPads como recurso didáctico e interactivo.

En $5^{\circ}$ de Primaria, concretamente en Matemáticas, se hizo una tarea de gamificación en grupo con las herramientas digitales de Quizizz y Makebadges para usar la división. Y en la asignatura de inglés se utilizó una aplicación pdi para una microactividad individual de relacionar conceptos y términos, además del libro digital para realizar speaking and listening.

En $4^{\circ}$ de Primaria, en la primera sesión de proyectos, concretamente en el ThePhotographer, se empleó la herramienta Popplet para hacer un mapa de ideas, extraídas de un tráiler en YouTube, hacerle fotos y subirlo a Google Classroom; en la segunda sesión, la actividad planteada fue buscar información sobre una de las islas, aunque no se llegó a implementar por la distracción del alumnado, volviendo a usar la aplicación LeoBien. 
En los proyectos Themuseums y LifeonEarth, de $5^{\circ}$, se realizaron actividades en grupo de búsqueda de información para la elaboración de un tríptico, sobre un museo, y para la realización de un mural.

En estas sesiones se han usado libros digitales y métodos didácticos digitales, como Google Classroom, combinados con otras herramientas como Kahoot, Quizizz, Makebadges, Popplet, Green Screen, TouchCast, etc. Se puede concluir que el profesorado emplea recursos didácticos digitales propios o sugiere al alumnado usar aplicaciones y materiales creados por otros, como en los proyectos.

En el Caso 2 (Galicia), la primera área de conocimiento en la que más se visibilizó ese proceso de digitalización y supresión de los libros de texto impresos fue la de matemáticas. Fueron retirando el uso del libro de texto y creando, mediante eXelearning, los temarios y contenidos curriculares en un aula virtual. Para hacerlo más motivador, dinamizaron un proyecto de Robótica para ampliar y afianzar los contenidos curriculares. "Las herramientas más utilizadas en estas aulas son: los robots y técnicas de programación en entornos gráficos editables como Scratch y mBlock, el escritorio virtual Netvives, donde se guarda y comparte todo tipo de recursos y herramientas digitales de autor para generar recursos como Jclic, EdiLimK, Kahoot, eXeLearning o Cubu, y alojarlos en su propio banco de recursos" (Galicia, coordinador TIC).

En Ciencias Sociales, el maestro comenta que apoya sus estrategias docentes en los libros digitales del proyecto Edixgal, la plataforma EVA, el Wordy aplicaciones de mapas conceptuales, juegos de trivial, rompecabezas, etc. La maestra de Lengua Española añade que también utilizan las TIC para realizar pruebas de examen y lecturas grupales.

Otra estrategia destacable es la de crear historias digitales inventadas sobre temas de diversidad, educación especial y /o actualidad. Esta actividad la realizan en sexto a través del Google Drive, porque permite retroalimentar, compartir y dar continuidad a una historia de interés común. Usan la hora de leer para subir información sobre la que van construyendo una historia, que se inicia en un aula y se finaliza en otra. Abordan temáticas controvertidas para integrarlas en cualquier dinámica de clase y desde el marco contextualizado del Ilamado "Programa TEI (Tutoría Entre Iguales) o del Plan de Orientación" (Galicia, maestra de 69.

En el caso de inglés, la maestra nos comenta que utiliza su blog y aplicaciones de acceso libre tales como Anki, Scratch, Voki, Scratch, Google Drive, WordReference, LibreOffice y para crear flashcards, reeditar canciones y/o repasar vocabulario (Galicia, maestra de inglés). 
Utilizan aplicaciones para buscar información (Google, wikis, blogs, LibreOffice Impress, etc.), escuchar música e idiomas (mBlock, English Blog, QuickSIS, Anki, etc.), editar textos, vídeos, juegos e imágenes (Dropbox, Scratch, Filmigo Video Maker, etc.) o elaborar tareas y trabajos académicos (Word, Pdf, PowerPoint, Prezti, Knovio, Excell, etc.). Para este equipo docente las TIC se convierten en unas herramientas didácticas eficaces cuando se logra una verdadera estrategia e innovación educativa.

En el Caso 3 (Valencia) de las estrategias docentes implementadas en las aulas de $5^{\circ}$ y $6^{\circ}$ de Primaria destacamos: el trabajar con materiales de elaboración propia y/o de licencia libre para atender a la diversidad del aula; Uso de plataformas gratuitas: Classroom (Thatquiz, Kahoot, etc.); Trabajo grupal: elaboración y exposición del alumnado de temas estudiados; Grupos de discusión; Diseños experimentales con proyectos de aula/Centro; Clases magistrales del docente; Publicación en el blog de tareas y trabajos realizados.

Todas estas estrategias sitúan al alumnado en el centro del proceso de enseñanza-aprendizaje dándole oportunidades de confrontar ideas, experimentar y aprender haciendo (Dewey, 2004). Pueden "crear y consumir materiales de las plataformas a través de actividades interactivas/vídeos" (Valencia, maestra de $6^{\circ}$ ).

Cada uno de los grupos-clase tiene su propio blog. Por ejemplo, el Blog Explosiu de $5^{\circ}$, que funciona como espacio de interacción entre alumnado y docente, como mural didáctico para compartir los proyectos realizados y constituye un espacio colaborativo para crear y publicar contenidos. Asimismo, es un medio de comunicación entre escuela y familia. Por tanto, es una herramienta que facilita la adquisición de la competencia digital y fomenta el desarrollo del pensamiento creativo, crítico, expresión de ideas y resolución de problemas. Observamos que el alumnado valora positivamente el uso del blog.

Las docentes destacan que utilizan la imagen como un elemento pedagógico relevante (Bautista García-Vera, 2019). "Lo justifican por la necesidad de superar el libro de texto tradicional pese a que es un trabajo interminable" (Valencia, maestra de 69. Los estudiantes del siglo XXI "son nativos digitales por lo que utilizar solo un libro de texto en papel, es perder posibilidades" (Valencia, maestra de 59. Además, enfatizan que los MDD facilitan la comunicación con el alumnado y la familia. Destacan que "con las Tabletas se facilita que los discentes trabajen en grupos y provoca el que se ayuden para realizar tareas. Las TIC potencian situaciones de aprendizaje para dialogar, cooperar, etc. Y eso facilita situaciones adecuadas de interacción en el aula de Primaria" (Valencia, maestra de 59. Consideran que una forma de socializar y construir el conocimiento en el aula es interaccionando 
y experimentando. Las docentes también admiten que se generan algunas dificultades en la implementación, aunque destacan que son más los beneficios que aportan las TIC que los inconvenientes que acarrean.

\section{Conclusiones}

Partiendo del problema inicial planteado - cómo la creación y usos de MDD, por parte de los docentes, potencia un cambio metodológico en las aulas de cuarto, quinto y sexto de Primaria-, asumimos que el entorno digital educativo es un espacio en continua evolución, donde la escuela del siglo XXI no debe ni puede quedarse atrás. Estamos ante una nueva reconversión digital de docentes, espacios y currículos (INTEF, 2017; UNESCO, 2008). La actual educación se conecta con la capacidad de seleccionar información (Southwell, 2013). La transformación de información en conocimiento es un proceso complejo en el que hemos de seguir investigando. Precisamente, este trabajo nos ha permitido destacar unas evidencias comunes en los tres casos:

- Son centros cuyas aulas ya han sustituido el uso del libro de texto impreso por la integración de los MDD.

- Enseñan mediante libros de texto digitales, en plataformas y con proyectos promovidos por sus pertinentes consejerías de educación, así como MDD de elaboración propia y diversa autoría que comparten a través de redes, bancos de recursos, blogs y espacios virtuales.

- Defienden que el uso de los MDD debe ser entendido como recursos a disposición del proceso de enseñanza-aprendizaje, para potenciar aprendizajes situados y relevantes.

- Consideran fundamental utilizar los MDD en la escuela, porque vivimos en una sociedad cada vez más globalizada y en donde la información y la comunicación son imprescindibles para desarrollarse como sujetos críticos y creativos.

- Muestran una gran preocupación por la formación del profesorado y por mantener la colaboración y coordinación entre profesorado, alumnado y familias.

- Entre los aspectos que sería necesario mejorar podríamos indicar:

- Ampliar las dotaciones económicas para recursos TIC en todas las aulas y espacios de los centros.

- Proceder a la actualización y dinamización del Plan TIC. 
- Ofrecer más formación pedagógica al profesorado y asesoramiento a las familias sobre el buen uso de las TIC y MDD en el centro y el hogar -la ciberseguridad-.

- Mejorar la formación del alumnado en un uso responsable de las tecnologías, así como en un uso más pedagógico para la búsqueda de información y posterior generación de conocimientos.

\section{Reconocimientos}

Este artículo forma parte de los diferentes estudios de campo realizados para el proyecto de investigación titulado "La escuela de la sociedad digital: análisis y propuestas para la producción y uso de los contenidos digitales educativos» (EDU2015-64593-R). Financiado por el Programa Estatal de I+D+i Orientada a los Retos de la Sociedad convocado por el Gobierno de España.

Agradecimientos, en especial, a los centros educativos que han participado y permitido realizar este trabajo en Canarias, Galicia y Valencia.

\section{Referencias}

Angrosino, M. (2012). Etnografía y observación participante en Investigación Cualitativa. Madrid: Morata.

Area, M. (2017). La metamorfosis digital del material didáctico tras el paréntesis Gutenberg. RELATEC, Revista Latinoamericana de Tecnología Educativa, 16(2), 13-28. https:// doi.org/10.17398/1695-288X.16.2.13

Area, M. (2020).Escuel@ Digit@I. Los materiales didácticos en la Red. Barcelona: Graó

Area, M., Parcerisa, A. y Rodríguez, J. (2010). Políticas educativas y buenas prácticas con TIC. Barcelona: Graó.

Arboleya, E. y Dopico, E. (2017). Superando las barreras físicas del aula: recursos naturales y TIC. Revista Iberoamericana de Educación, 75(1), 71-88. https://doi.org/10.35362/ rie7511356

Bautista, M.G., Martínez, A.R. e Hiracheta, R. (2014). El uso de material didáctico y las tecnologías de información y comunicación para mejorar el alcance académico. Recuperado de https://bit.ly/37qktXA

Bautista García-Vera, A. (Coord.) (2019). La fotografía en la Formación del Profesorado. Madrid: Narcea. 
Cepeda, O., Gallardo, I.M. y Rodríguez, J. (2017). La evaluación de los materiales didácticos digitales. RELATEC, Revista Latinoamericana de Tecnología Educativa, 16(2), 79 95. https://doi.org/10.17398/1695-288X.16.2.79

De Pablos, J. (Coord.). (2015). Los centros educativos ante el desafío de las tecnologías digitales. Madrid: La Muralla.

Dewey, J. (2004). Democracia y educación, una introducción a la filosofía de John Dewey. Madrid: Morata.

Duffy, T.M. y Jonassen, D.H. (2013). Constructivism and the technology of instruction: a conversation. New York: Routledge.

Flick, U. (2004). El diseño de la investigación cualitativa. Madrid: Morata.

Freire, P. (1997). Pedagogía de la Autonomía. México: Siglo XXI.

García-Martín, S. y Cantón-Mayo, I. (2019). Use of technologies and academic performance in adolescent students. Comunicar, 59, 73-81. https://doi.org/10.3916/C59-2019-07

George, C. (2020). Alfabetización y alfabetización digital. Transdigital 1(1), 1-17. Recuperado de https://bit.ly/39tJzYi

González C. J., Martín, S. y Vega, A. (2018). Portales educativos: la producción de materiales didácticos digitales, @tic. Revista d'innovació educativa, 20, 89-97. http://doi. org/10.7203/attic.20.12139

INTEF (2017). Marco Común de Competencia Digital. Recuperado de https://goo.gl/ R9ia89

Ley Orgánica 8/2013, de 9 de diciembre, para la Mejora de la Calidad Educativa. (LOMCE). Boletín Oficial del Estado, 295, 97858-97920. Recuperado de https://bit.ly/39srSbm

López-Roldán, P. y Fachelli, S. (2015). Metodología de la investigación social cuantitativa. Barcelona: UAB

Moliner, M. (1998). Diccionario del uso del español. Madrid: Gredos.

Peirats, J., Gallardo, I.M.; San Martín, Á. y Cortés, S. (2015). Los contenidos curriculares digitalizados: Voces y silencios en el ámbito editorial. Educatio Siglo XXI, 33(3), 39-62. https://doi.org/10.6018/j/240801

Pérez-Gómez, A. (2012). Educarse en la era digital. Madrid: Morata.

Proyecto Abalar (2013). Estrategia para la integración plena de las TIC en la práctica educativa de Galicia. Recuperado de https://bit.ly/3lobTOp

Resolución de 10 de junio de 2013, de la Dirección General de Innovación, Ordenación y Política Lingüística, por la que se convoca un Programa experimental para el fomento del uso de libros de texto en dispositivos electrónicos denominados tabletas en centros educativos sostenidos con fondos públicos de la Comunitat Valenciana (DOCV, 7047, 17/06/2013). Recuperado de https://bit.ly/39mLVbk

San Martín, Á. y Peirats, J. (2018). Controversias en la transición del libro de texto en papel y electrónico a los contenidos digitales. RED, Revista de Educación a Distancia, 56, 1-17. Recuperado de http://bit.ly/2W40ciM

Southwell, M. (2013). La escuela ante nuevos desafíos: participación, ciudadanía y nuevas alfabetizaciones. Buenos Aires: Santillana. 
Stake, R. (1998). Investigación con estudio de casos. Madrid: Morata.

Simons, H. (2011). El estudio de caso: teoría y práctica. Madrid: Morata.

Taylor, S.J. y Bogdan, R.C. (1984). Introducción a los métodos cualitativos de investigación. Barcelona: Paidós.

UNESCO (2008). Estándares de Competencia en TIC para docentes. París: UNESCO. Recuperado de https://bit.ly/3fRD5TW

Vidal, M.I., Vega, A. y López, S. (2019). Uso de materiales didácticos digitales en las aulas de Primaria. Campus Virtuales, 8(2), 103-119. Recuperado de https://bit.ly/3fNGFOY

Weller, M. (2016). The Open Flip - A Digital Economic Model for Education. Journal of Learning for Development, 3(2), 26-34. Recuperado de https://bit.ly/3ocOJvB 